\title{
Discriminación salarial de la mujer en el mercado laboral de México y sus regiones
}

\section{Gender wage discrimination in the labor market in Mexico and its regions}

\author{
Reyna Elizabeth Rodríguez-Pérez \\ David Castro-Lugo*
}

\begin{abstract}
The goal of this paper is to analyze gender job earnings, determine the wage gap and discrimination in Mexico and its regions. We apply the Oaxaca-Blinder decomposition to the 2000-2004 National Urban Employment Survey 20002004. We found a gender wage gap in favor of men, and more than 85 percent of these differences are linked to discrimination. Regionally, the gap oscillates between 15 and $25 \%$ and the discrimination tends to converge at 20\%, however, in the Capital region it converges at 13 por ciento.
\end{abstract}

Keywords: discrimination, wage inequity, Mexico, labor market, regions.

\begin{abstract}
Resumen
El objetivo de este artículo es analizar las remuneraciones salariales por sexo, determinar la brecha y discriminación que existe en México y regiones con respecto a la materia, empleando la descomposición Oaxaca-Blinder y la Encuesta Nacional de Empleo Urbano (ENEU) 2000-2004. Los resultados indican que existen diferencias salariales por sexo a favor de los hombres y más de $85 \%$ de estas desigualdades se vinculan con la discriminación. Por regiones existe un comportamiento heterogéneo con oscilaciones entre 15 y $25 \%$ y discriminación que tiende a converger en $20 \%$, con excepción de la capital que se ubica en 13 por ciento.
\end{abstract}

Palabras clave: discriminación, desigualdad salarial, México, mercado laboral, regiones.

*Universidad Autónoma de Coahuila. Correos-e: reynarodriguez@uadec.edu.mx, david.castro@ uadec.edu.mx 


\section{Introducción}

Cuando se analiza el mercado laboral de cualquier delimitación geográfica se distinguen diversas ocupaciones y, en correspondencia, también distintas remuneraciones por cada tipo de trabajo. En un mercado competitivo estas diferencias en las retribuciones encuentran sustento en las condiciones de oferta y demanda, así como en los niveles de productividad, vinculados fundamentalmente con la dotación de capital humano, donde si los elementos asociados a la condición religiosa, color de piel, sexo, etc., no constituyen un factor que incida sobre la productividad, no deben reflejar diferencias en las remuneraciones.

Esta relación no parece ser coherente para las mujeres, ya que aunque en las últimas décadas han incrementado su nivel de educación y su inserción en el mercado laboral, existe desigualdad salarial por sexo. De esta manera, Rendón (2003) asevera que dicha desigualdad es producto de una construcción social, conocida como el sistema sexolgénero. Donde el género constituye una concepción creada a partir de las diferencias de sexo que distingue culturalmente a las mujeres de los hombres y les atribuye características diferentes.

Algunas investigaciones demuestran que existe desigualdad salarial por sexo en el mercado laboral; sin embargo, sólo parte de ellas se han abocado a analizar sus causas. Una de las explicaciones sobre este comportamiento se basa en la discriminación por género, que, a su vez, se define como un fenómeno en el que la mujer es remunerada de forma diferente a causa de prejuicios y no por su productividad.

Esto ha generado una gran carga de juicios que se traducen en la hipótesis sobre discriminación de la mujer en el mercado de trabajo, donde la selectividad, de acuerdo con la teoría del capital humano, debería ser solamente por diferencia de grados de calificación. Por lo tanto, esto nos lleva a incursionar en el análisis de la desigualdad salarial que permita determinar si tales diferencias se deben a las dotaciones de capital humano (características) o por discriminación salarial (retribución a los factores).

El objetivo de este artículo es analizar las remuneraciones salariales por sexo para determinar sus diferencias y, mediante su descomposición, estimar el porcentaje de discriminación salarial en México (capital) y regiones. Para ello se aplica la técnica Oaxaca-Blinder utilizando como fuente de información la Encuesta Nacional de Empleo Urbano (ENEU) del 2000 al 2004.

Incorporando las características demográficas y ocupaciones, los resultados indican que en el periodo analizado, a nivel nacional, existen diferencias salariales por sexo a favor de los hombres del orden de $20 \%$ y que más de $85 \%$ de éstas están asociadas con la discriminación, dado que 
son desigualdades no atribuibles a las dotaciones de capital humano. Por regiones se aprecia un comportamiento heterogéneo donde algunas desigualdades oscilan entre 15 y $25 \%$, con niveles de discriminación que tienden a converger en $20 \%$, con excepción de la región capital que se ubica sobre 13 por ciento.

En este artículo se exponen diferentes conceptualizaciones de la discriminación, en el primer apartado, posteriormente se hace una revisión de la evidencia empírica con respecto al tema; mientras que en la siguiente sección se presentan los datos a utilizar, así como la metodología, es decir, se realiza una exploración de las características del mercado laboral femenino destacando aspectos de estructura ocupacional y remuneraciones $\mathrm{y}$, finalmente, se plantean las estimaciones e interpretación de resultados.

\section{Discusión sobre la teoría de la discriminación salarial}

Frecuentemente escuchamos hablar de discriminación, sin embargo, puede no ser tarea fácil dar una definición sobre la misma; al respecto Becker (1971: 13) establece que desde la perspectiva sociopsicológica "se dice que una persona discrimina en contra (o a favor) de otra si el comportamiento de ésta última no está motivada por una consideración 'objetiva'”.

El mismo autor establece que esta definición presenta el inconveniente de que es difícil identificar una violación de hechos objetivos sobre aquellos relacionados con gustos o valores, por ejemplo: ¿se puede hablar de discriminación por el hecho de que alguien prefiera el tipo de ropa, peinado o calzado que utiliza una persona famosa a la de cualquier otra?

Sin el objetivo de llevar a cabo una discusión filosófica sobre el término, dado que supera las pretensiones del presente artículo, se pretende mostrar la dificultad para definir un concepto general de discriminación y para ser más preciso es necesario asignar un apellido a la misma o circunscribirla a una disciplina; así, mientras para los sociólogos la discriminación en contra de un grupo en particular puede estar asociada con la distancia de este grupo respecto a un determinado estatus social, para los psicólogos este mismo término puede implicar clasificar a los individuos de acuerdo con sus características de personalidad (Becker, 1971).

Desde el punto de vista económico puede abordarse esta concepción al retomar la idea de Becker (1971), considerando que todas las personas que contribuyen en la misma magnitud a la producción pueden conformarse en un grupo, y cada uno constituye un factor de producción que presenta distintos niveles de productividad asociados con sus características particulares.

Derivado de lo anterior, Autor (2003: 1) define discriminación "cuando miembros de una minoría son tratados diferencialmente (menos fa- 
vorablemente) que los miembros de un grupo mayoritario con idénticas características productivas".

Una definición más puntual es la que aportan McConnell et al. (2003: 435) quienes establecen que "Existe discriminación económica cuando las mujeres o los trabajadores pertenecientes a grupos minoritarios -que tienen la misma capacidad, nivel de estudios, formación y experiencia que los varones del grupo mayoritario- reciben un trato inferior en la contratación, el acceso a una ocupación, los ascensos, el salario o las condiciones de trabajo".

La discriminación también puede adoptar la forma de desigualdad en el acceso a la educación, el aprendizaje o los programas de formación en el trabajo, el cual tiene que ver con el aumento del stock de capital humano de las personas.

A partir de esta definición se puede establecer que dentro del mercado laboral existen diferentes tipos de discriminación, como puede ser la ocupacional (acceso a una determinada ocupación), en el empleo (contratación), en la formación (educación) y salarial.

Sin dejar de reconocer la relevancia de los otros elementos y de estar conscientes de que la presencia de un determinado tipo de discriminación afecta o incide sobre el resto, ${ }^{1}$ en este documento abordamos la discriminación salarial, partiendo de la consideración de competencia perfecta en el mercado de trabajo.

La concepción de discriminación salarial es cuando "las mujeres ganan menos que los hombres por hacer el mismo trabajo. En términos más técnicos, existe discriminación salarial cuando las diferencias salariales no se deben a la presencia de diferencias en productividad" McConnell et al. (2003: 435); concepto que es compartido por Di Paola y Berges (1997) ya que mencionan que existe discriminación cuando el mercado ofrece distintas oportunidades a las personas similares que sólo se diferencian por su raza, grupo étnico, género, edad u otras características personales.

Esta investigación se centra en explorar la presencia de discriminación salarial por género en México y regiones para el periodo 2000-2004 considerando como fuente de información los microdatos disponibles de la ENEU.

Cuando se analizan las diferencias salariales por sexo, un punto importante que a veces no es tomado en cuenta, es que el trabajo de la mujer involucra un costo de oportunidad mayor al que puede tener cualquier hombre, debido al valor que atribuye a otras actividades productivas no remuneradas tales como el cuidado del hogar, tareas de ama

\footnotetext{
${ }^{1}$ Como puede ser el hecho de que la discriminación en el empleo afecta la tasas de desocupación femenina y ésta a su vez impacta sobre las remuneraciones de las mujeres.
} 
de casa, crianza de los hijos, sobre todo en los primeros ańos de vida y hasta que alcanzan la edad escolar. Tales actividades han dado origen a la segregación ocupacional por sexo, que no es otra cosa que la división del trabajo extradoméstico entre dos grupos sociales, que provoca que se desperdicie el capital humano y los niveles de ingreso sean reducidos en el mercado laboral para las mujeres.

En el campo de la discriminación laboral existen varias teorías que intentan explicar la presencia de ésta, entre las que destacan: 1) el modelo del gusto por la discriminación; 2) la discriminación estadística y, 3) el modelo de concentración, mejor conocido como de segregación ocupacional.

Mientras las dos primeras se ubican dentro de lo que se denomina discriminación posmercado, dado que ocurre una vez que los individuos se encuentran dentro del mercado laboral, la última se ubica en la condición de discriminación premercado, debido que se presenta antes de que participe en el mercado laboral.

Dado nuestro objetivo de estudio y fuentes de información utilizadas, el análisis se ubica dentro de la primera categoría, reconociendo que no obstante la relevancia y el efecto que esta última puede tener sobre los resultados finales que obtengamos. Este tipo de discriminación no será abordada en el documento.

La teoría del gusto por la discriminación fue desarrollada por Becker $(1971)^{2}$ y expone que la discriminación parte del gusto de las personas por segregar a otras, y los factores que pueden estar detrás de este comportamiento son diversos; este gusto lleva al individuo a actuar como si tuviera una disponibilidad a pagar para evitar realizar transacciones con las personas desfavorecidas, dado que ello le implica un costo subjetivo o psicológico.

Aun cuando esta conducta puede ser practicada por distintos agentes como consumidores y productores, en el caso de estos últimos, por ejemplo, su gusto por la discriminación puede llevarlos a contratar varones a un precio mayor con el fin de no emplear a mujeres aun cuando ambos son igualmente productivos o, en su caso, contratar a mujeres con remuneraciones inferiores a la que reciben los varones.

Para determinar la magnitud del gusto por la discriminación Becker (1971) midió la discriminación por medio de un coeficiente $d$ que refleja el impacto de los costos discriminatorios en términos monetarios y permite actuar como un puente entre costo monetario y costo neto. Este coeficiente se explica de la siguiente manera: Si el empleador no tiene prejuicios para contratar a hombres, el costo de contratarlo es el salario

${ }^{2}$ Una primera edición de este documento es de 1957. 
$W_{h}$; Sin embargo, el costo de emplear a una mujer sería el salario $W_{m}$, más el costo subjetivo.

Por tanto, el empresario que tiene prejuicios sólo contratará mujeres si su salario es inferior al de los hombres, así, para emplear mujeres, el salario debe ser menor que el de los varones en la cuantía del coeficiente de discriminación, que no es otra cosa que el valor monetario de su costo subjetivo, así $W_{h}=W_{h}-\mathrm{d}$. $^{3}$

Cuanto mayor sea el gusto por la discriminación, reflejado en el valor $d$, mayor será la disparidad entre los salarios de los hombres y las mujeres para que el empresario decida contratar a estas últimas.

Una de las derivaciones de esta teoría es que los empleadores que tienen gusto por la discriminación incurrirían en ineficiencias económicas y desventaja competitiva frente a los no discriminadores, dado que estos últimos pueden contratar personal igualmente productivo a menor costo.

Este elemento establece que en presencia de mercados competitivos, las empresas discriminadoras saldrían del mercado o perderían cuota de mercado, lo que llevaría a una reducción de la discriminación; por tanto, si queremos combatir este fenómeno sólo hay que fomentar la existencia de mercados competitivos. ${ }^{4}$

La persistencia de las diferencias salariales por sexo, no obstante que se han desarrollado esquemas para hacer los mercados más competitivos, llevaron a plantear otras teorías para dar respuesta a este fenómeno, dando origen al argumento de la discriminación estadística que parte de la idea de que se juzga a las personas a partir de las características promedio del grupo al que pertenece y no de las cualidades individuales.

Así, cuanto más heterogéneo sea el grupo más diferencias existirán entre el promedio y cada individuo, y por tanto, mayor será el nivel de error y la posibilidad de discriminación por este hecho, por ejemplo, si consideramos al grupo de mujeres cuyas características laborales son: su participación en el mercado de trabajo es intermitente por la etapa reproductiva, que asignan una gran prioridad al cuidado de los hijos, que desearían jornadas laborales más cortas, trabajos próximos al hogar, menor disponibilidad para viajar y realizar horas extras, etc.; una oferta laboral que no se apegue a estas características seguramente no será asignada a una mujer, aun cuando una de las aspirantes a este puesto cumpla con los requerimientos que se necesitarían y sus características fueran distintas al promedio estadístico de su grupo.

\footnotetext{
${ }^{3}$ Para mayor detalle sobre la teoría del gusto por la discriminación véase Becker (1971).

${ }^{4}$ Esta hipótesis, derivada del trabajo de Becker (1971), supondría que a mayor apertura comercial o globalización la brecha salarial por género se reduciría. Dentro de los estudios que abordan esta perspectiva podemos mencionar a Artecona y Cunningham (2002); Menon y Van der Meulen (2009); El Hamidi (2008); Domínguez y Brown (2010) y Aguayo-Tellez (2011).
} 
A diferencia de lo que ocurre en la teoría anterior, en este caso puede decirse que no existe un gusto por la discriminación, sino que el empleador a partir de características como la edad, sexo, raza, etcétera intenta aproximar atributos de producción, apoyado en el comportamiento promedio, lo que implicaría la existencia de una racionalidad y no necesariamente una desventaja; además, bajo este argumento teórico no existen elementos que nos permitan suponer que la discriminación estadística vaya a disminuir en el tiempo (McConnell et al., 2003).

Blinder (1973) y Oaxaca (1973) proponen un modelo econométrico para medir la discriminación salarial, el cual se ha convertido en la herramienta más empleada en el área para realizar este tipo de estimaciones, ${ }^{5}$ de esta manera, descomponen la brecha salarial que se divide en dos partes: la primera contiene diferencias entre grupos asociadas al capital humano como la educación y la experiencia y la segunda, a las características inobservables.

Tales estimaciones permiten obtener una medida más exacta de la magnitud de la discriminación salarial, que la referida solamente a las diferencias salariales; no obstante, actualmente existen nuevas propuestas metodológicas que buscan medir la brecha salarial y sus componentes a lo largo de la distribución (Albrecht et al., 2009; Río et al., 2011).

\section{Evidencia empírica}

Una vez presentadas algunas definiciones y los principales argumentos teóricos que se han esgrimido para comprender la discriminación, en este apartado se expone una breve revisión sobre la evidencia empírica, considerando estudios que explican la desigualdad salarial por género y si ésta se debe a las diferencias en las características de capital humano o existe discriminación salarial.

El estudio de la brecha salarial y discriminación ha sido abordado en una diversidad de países, ${ }^{6}$ España ha sido objeto de varios estudios, dentro de los cuales se pueden mencionar: Hernández-Martínez (1995); Rica y Ugidos (1995); Ullibarri-Arce (2003); Ramos et al. (2002); García y Morales (2006); Rica et al. (2008) y Río et al. (2011), por citar algunos. Los estudios van desde 1987 hasta 2003, en los cuales se identifican dos principales enfoques metodológicos: aquellos que utilizan el método tradicional (Oaxaca-Blinder o alguna de sus variantes), ${ }^{7}$ mismos que centran su análisis en la media de la distribución (primeros seis estudios

${ }^{5}$ Lo que la ha llevado a considerarla como la metodología tradicional.

${ }^{6} \mathrm{Al}$ respecto véase Weichselbaumer y Winter-Ebmer (2005).

7 Para una discusión sobre algunas propuestas alternativas y posibles efectos véase Oaxaca y Ranson (1994), y Silber y Weber (1999). 
citados), y métodos alternativos que analizan la totalidad de distribución (últimos dos artículos).

Los resultados, bajo diferentes enfoques metodológicos y años, indican la presencia de brecha salarial en contra de las mujeres y que una proporción importante de la misma no se explica por las características, lo que puede interpretarse como discriminación.

En los estudios realizados en Mar del Plata, Argentina, destacan los autores Di Paola y Berges (1997), quienes utilizan la Encuesta Permanente de Hogares (EPH) para los años 1993 y 1997, y Di Pasquale y Atucha (2003), quienes en sus estudios del 2000 también encuentran la existencia de brecha salarial, y mediante métodos de descomposición vinculados al valor medio de la distribución, identificaron que aproximadamente dos terceras partes de las diferencias no son explicadas por factores asociados con las características.

China es una de las naciones que ha despertado mayor interés sobre este tema, por los efectos que la transformación económica puede ejercer sobre la disparidad salarial por género; al respecto Chi et al. (2007) realiza un estudio cuyo periodo va de 1987 a 2004 a partir de la Encuesta de Hogares Urbanos (EHU), considerando niveles educativos, sector económico, ocupación y regiones. Sus resultados indican que durante el periodo analizado se amplió la brecha salarial, ocasionada por un incremento en las diferencias entre individuos con estudios universitarios en la industria y entre regiones; ${ }^{8}$ comportamiento diferente al observado para el caso de Chile, donde Fuentes et al. (2005) analiza la discriminación salarial por género para los años 1990 y 2003 y encuentra que ésta ha disminuido, con lo que para el último año, la diferencia no explicada representa sólo 27.5 por ciento. ${ }^{9}$

Dentro de los trabajos sobre brecha salarial y discriminación realizados para el caso de México se pueden mencionar: Alarcón y McKinley (1994), Garro-Bordonaro y Rodríguez-Oreggia (1995), Brown et al. (1999), Artecona-Cunningham (2002), Martínez-Jasso y Acevedo-Flores (2004), Pagan y Ullibarri (2000), Rodríguez-Pérez y Camberos-Castro (2007), Brown y Domínguez (2007), Cacciamali y Tatei (2013), RodríguezPérez y Castro-Lugo (2014), Castro et al. (2014), Popli (2008, 2013), y Arceo y Campos (2013), entre otros.

\footnotetext{
${ }^{8}$ Cheng et al. (2013) explora y confirma la presencia de discriminación salarial para los migrantes rurales frente a los trabajadores locales, lo que constituye una vertiente de análisis relevante donde se presentan flujos migratorios importantes.

${ }^{9}$ Montero y Garcés (2009) documentan la presencia de discriminación salarial en contra de la población indígena de Chile para el periodo 1996-2006.
} 
Estos estudios cubren desde la segunda mitad de los ochenta hasta 2011, considerando diferentes fuentes de información, ${ }^{10}$ así como distintos enfoques metodológicos, destacando el tradicional como el más frecuente, no obstante en años recientes se han explorado enfoques alternativos para descomponer la brecha salarial, buscando identificar algunos fenómenos en el comportamiento de las diferencias de ingreso por género a lo largo de la distribución, como pueden ser la presencia techo de cristal y piso pegajoso. ${ }^{11}$

Dentro de los principales resultados que destacan a lo largo de estas décadas de estudio es que una proporción importante de la brecha salarial por sexo no puede ser explicada por las dotaciones de factores; un menor consenso en la magnitud de la brecha salarial, aunque en general parece existir una tendencia hacia una reducción de ésta y una insuficiencia de estudios que aborden el aspecto regional, el cual es de suma relevancia en un país donde la heterogeneidad en tales demarcaciones constituyen una de sus principales características, por ello es importante la realización de estudios que cubran este aspecto.

\section{Datos y método empleado}

\subsection{Bases de datos empleadas}

Las bases de datos utilizadas en esta investigación corresponden a la ENEU 2000-2004. Dichas encuestas se homogenizan considerando las áreas metropolitanas contenidas en la ENEU 2004, ya que son las que se mantienen para todo el periodo analizado y que sumaron 32 ciudades. La razón para utilizar este periodo se fundamenta en dos aspectos relevantes: el primero vinculado con la permanencia de la ENEU hasta el último año de referencia ${ }^{12}$ y, el segundo, porque no existe información sobre el comportamiento de la discriminación en este periodo.

Se emplea la regionalización del territorio mexicano sugerida por Hanson (2003) que divide al país en seis regiones, la razón para tomar esta clasificación del territorio es que permite hacer una distinción entre estados que en principio presentarían diferentes efectos a causa de la

\footnotetext{
${ }^{10}$ Encuesta Nacional de Empleo Urbano (ENEU), Encuesta Nacional de Ocupación y Empleo (ENOE), Encuesta Nacional de Ingreso Gasto de los Hogares (ENIGH), Encuesta Nacional de Empleo, Salarios, Tecnología y Capacitación (ENESTyc) y Censos Generales de Población y Vivienda.

${ }^{11}$ Por techo de cristal se entiende cuando la brecha salarial por sexo, a favor de los hombres, se incrementa en la parte superior de la distribución, indicando la existencia de un tope o techo de ingresos para las mujeres y, por piso pegajoso se entiende la presencia de una brecha salarial significativamente alta en la parte inferior de la distribución.

${ }^{12}$ A partir de 2005 la ENEu es sustituida por la Encuesta Nacional de Ocupación y Empleo (ENOE), que aun cuando tiene objetivos que pueden considerarse similares, presenta modificaciones en su estructura, lo que puede ocasionar alteraciones en la comparabilidad de resultados.
} 
apertura comercial, y cómo esto afecta al mercado laboral regional, en particular, al respecto de la discriminación salarial, estos elementos permitirían distinguir esta investigación del resto, dado que no se han hecho estudios previos para este periodo ni se ha abordado el tema regional sobre este tema.

La población objetivo es el personal ocupado de 16 a 65 años, con jornada laboral semanal entre 20 y 55 horas. Los salarios de los trabajadores son captados por la encuesta de forma mensual en moneda corriente. En el trabajo empírico se calculan los salarios a precios de 2000 referidos al segundo trimestre, deflactados con el índice de precios al consumidor por estratos de salario del Banco de México y divididos su valor por el tipo de cambio promedio pesos por dólar en el mismo trimestre. Los trabajadores son clasificados en seis niveles de escolaridad, 18 ocupaciones y nueve actividades económicas.

El cuadro 1 presenta las principales características de los datos, donde se puede señalar que cada año aporta aproximadamente $20 \%$ de la muestra, la participación a nivel espacial indica que existe una mayor concentración de trabajadores en la capital del país con un porcentaje de $43.1 \%$, mientras que la región sur experimenta la menor participación con $3.3 \%$; también se registra la información de las características personales (sexo, estado civil, escolaridad) y laborales (sector de actividad, ocupación).

Donde se destacan los siguientes datos: el salario mensual promedio en el periodo 2000-2004 fue de 435.6 dólares mensuales; la participación promedio de las mujeres en el periodo de análisis se ubica en 39\%. La edad promedio de los trabajadores es de 34 años y aproximadamente la mitad reportan estar casados. La clasificación por nivel de escolaridad revela que la mayor parte de los ocupados asalariados cuentan con estudios de secundaria $(37 \%)$ y, en menor medida, se ubican los que tienen estudios de posgrado con $1.7 \%$, mientras que ocho de cada cien se encuentran sin instrucción escolar.

Las ocupaciones con mayor representación son las administrativas: artesanos, trabajadores fabriles y comerciantes; mientras que las actividades económicas más relevantes son la manufactura, servicios financieros, comercio y servicios diversos; por su parte, la actividad gubernamental participa con 9.6 por ciento. 


\section{Cuadro 1}

\section{Características generales de la información, 2000-2004}

\begin{tabular}{|c|c|c|c|}
\hline Variable & Cifras & Variable & Cifras \\
\hline $\begin{array}{l}\text { Salarios promedio } \\
\text { mensual }\end{array}$ & 435.6 usd & Técnicos & 5.81 \\
\hline Datos por año (\%) & 100.0 & Educación & 4.27 \\
\hline 2000 & 20.0 & Arte y Deportes & 0.87 \\
\hline 2001 & 20.2 & Funcionarios y directivos & 3.04 \\
\hline 2002 & 20.3 & Actividades primarias & 0.32 \\
\hline 2003 & 19.8 & $\begin{array}{l}\text { Jefes en la fabricación artesanal e } \\
\text { industrial }\end{array}$ & 3.28 \\
\hline 2004 & 19.3 & Artesanos y trabajadores fabriles & 11.34 \\
\hline Regiones (\%) & 100.0 & Operadores de maquinaria fija & 7.88 \\
\hline Frontera & 17.6 & $\begin{array}{l}\text { Ayudantes en fabricación artesanal } \\
\text { e industrial }\end{array}$ & 7.29 \\
\hline Norte & 7.6 & Conductores de maquinaria & 3.44 \\
\hline Centro & 23.0 & Jefes en actividades administrativas & 5.19 \\
\hline Capital & 43.1 & Administrativas & 14.58 \\
\hline Sur & 3.3 & Comerciantes & 9.58 \\
\hline Península & 5.3 & Vendedores ambulantes & 0.38 \\
\hline Jornada (horas semanales) & 45 & Servicios personales & 7.92 \\
\hline Sexo (masculino) (\%) & 61.0 & Servicios domésticos & 5.03 \\
\hline Edad (años) & 34 & Servicios de protección y vigilancia & 4.09 \\
\hline Estado civil (casado) \% & 48.0 & Actividad económica (\%) & 100.0 \\
\hline Escolaridad (\%) & 100.0 & Minería, electricidad, gas y agua & 1.3 \\
\hline Sin instrucción & 7.9 & Industria manufacturera & 24.6 \\
\hline Primaria & 20.2 & Construcción & 6.2 \\
\hline Secundaria & 37.0 & Comercio & 17.8 \\
\hline Preparatoria & 16.2 & Restaurantes y hoteles & 4.4 \\
\hline Universidad & 17.0 & Almacenamiento y comunicaciones & 4.2 \\
\hline Posgrado & 1.7 & Servicios financieros, seguros & 19.8 \\
\hline Ocupaciones (\%) & 100.0 & Servicios diversos & 12.1 \\
\hline Profesionistas & 5.69 & Gobierno & 9.6 \\
\hline
\end{tabular}

Fuente: elaboración propia a partir de ENEU, varios años. 


\subsection{Método de Blinder-Oaxaca para medir la discriminación salarial}

El modelo de la determinación de los salarios en capital humano se apoya en la ecuación desarrollada por Mincer (1974) para los datos individuales. En la función minceriana el logaritmo del ingreso se determina por los productos de cada variable independiente y los parámetros $\beta$ ya que éstos se aproximan a la tasa de rentabilidad más el término de perturbación aleatoria $\mu$.

$$
\operatorname{Iny}_{i}=\beta_{0}+\beta_{1} s_{i}+\beta_{2} x_{i}+\beta_{3} x_{i}^{2}+\mu_{i}
$$

La connotación $i$ se refiere al i-esimo individuo, $\operatorname{Iny}_{i}$ es el logaritmo natural del ingreso, $s_{i}$ es el nivel de educación formal alcanzado en número de años de educación, $x_{i}$ es la experiencia laboral del individuo medida $x=t-s-6, t$, es la edad del individuo, $s$ son los ańos de escolaridad menos seis, ya que es la edad en la que el individuo ingresa a su vida escolarizada, $x_{i}^{2}$ es el término cuadrático de la experiencia donde se captan los rendimientos decrecientes del capital humano y, finalmente, $\mu_{t}$ es el término de perturbación aleatoria que sigue una distribución normal con media cero y varianza constante.

Para completar esta investigación es importante conocer si las diferencias salariales se deben a las dotaciones de capital humano o por efecto de la discriminación salarial por género en el mercado de trabajo de México y regiones. El cálculo de la brecha de ingresos entre géneros se produce desde las funciones de ingreso mincerianas simples estimadas para ambos sexos, como se mencionó anteriormente, y la descomposición del diferencial de salarios con la técnica de Oaxaca (1973) y Blinder (1973).

La ausencia de discriminación supone que las remuneraciones generadas por las dotaciones de capital humano deben ser idénticos para ambos grupos; de forma que las diferencias en las retribuciones sólo reflejen disparidades en las dotaciones de capital humano y, por tanto, en la productividad laboral, de no ser así, las inequidades salariales podrían atribuirse a la discriminación.

Las ecuaciones salariales son estimadas de forma separada para hombres y mujeres y a partir de la regresión lineal, ésta pasa a través de las medias de las variables, por lo que las diferencias salariales por sexo se pueden expresar como:

$$
\operatorname{In}\left(w_{m}\right)-\operatorname{In}\left(w_{f}\right)=X_{m}^{\prime} b_{m}-X_{f}^{\prime} b_{f}
$$




\section{Donde:}

In $\left(w_{m}\right)$ y In $(w)$ son las medias del logaritmo natural de ingresos de hombres $(m)$ y mujeres $(f)$.

$X_{m}$ y $X_{m}$ son vectores que contienen las medias de las variables para hombres y mujeres, respectivamente, y $b_{m}$ y $b_{f}$ son los coeficientes estimados.

Proporcionado estos resultados, el logaritmo diferencial de ingresos puede ser descompuesto en dos vías. $\Delta X^{\prime}=X_{m}^{\prime}-X_{f}^{\prime}, \mathrm{y} \Delta=b_{m}$ y $b_{f}$, la ecuación anterior puede escribirse de la siguiente manera:

$$
\operatorname{In}\left(w_{m}\right)-\operatorname{In}\left(w_{f}\right)=\left(X_{m} b_{m}-X_{f}\right)^{\prime} \beta_{m}+X_{f}^{\prime}\left(\beta_{m}-\beta_{f}\right)
$$

El primer término es la parte de la disparidad del logaritmo de ingresos, debido a las diferencias (promedio) de las características entre hombres y mujeres, y el segundo término es la parte de la desigualdad asociado con la diferencia en las remuneraciones a las características, es decir, expresa la diversa estructura de salarios. En ausencia de discriminación, hombres y mujeres deberían de recibir igual salarios para los mismos atributos de capital humano, entonces las discrepancias en ingresos deberían ser sólo por diferencias con las características, por tanto, el segundo término podría estar interpretado como la parte de la diferencia del logaritmo del ingreso debido a la discriminación, es decir, es la parte de la desigualdad de ingreso no asociada a las dotaciones de factores. Esto es, en esencia, el enfoque de Oaxaca y Blinder, tal como lo refiere Neumark (1988).

\section{Características del mercado laboral femenino y diferencial de salarios}

\subsection{Las mujeres en el mercado laboral: estructura y remuneraciones}

Una de las características más relevantes del mercado laboral en las últimas décadas es la creciente participación de las mujeres dentro de la población económicamente activa; así, mientras en 1970 la tasa de participación era de $17.6 \%$, en 2008 pasó a 42\% (INEGI, 2009).

Los factores que están detrás de dicho crecimiento son de diferente naturaleza y responden tanto a elementos que pueden considerarse coyunturales como estructurales, dentro de los cuales podemos mencionar: mayor nivel de educación femenino, incrementos en la productividad doméstica, reducción del número de hijos, creciente sustitución de bienes 
y servicios elaborados en el hogar por el mercado, desarrollo de actividades que permiten una mayor presencia de mujeres, cambios culturales, transformaciones en el modelo familiar organizativo, caída del poder adquisitivo de los salarios, etcétera. ${ }^{13}$

La información utilizada para este análisis se presenta en el cuadro 2, y aunque puede considerarse como un periodo breve, permite exponer dicho fenómeno, donde se destaca una tendencia creciente de la participación femenina en el mercado laboral.

\section{Cuadro 2}

Estructura ocupacional por sexo, 2000-2004

\begin{tabular}{lccc}
\hline \multicolumn{1}{c}{ Año } & Hombres & Mujeres & Total \\
\hline 2000 & 61.8 & 38.2 & 100.0 \\
2001 & 61.0 & 39.0 & 100.0 \\
2002 & 61.1 & 38.9 & 100.0 \\
2003 & 61.4 & 38.6 & 100.0 \\
2004 & 60.6 & 39.4 & 100.0 \\
\hline
\end{tabular}

Nota: información considerando las 32 ciudades o áreas metropolitanas.

Fuente: elaboración propia a partir de ENEU, varios ańos.

¿Esta creciente participación se refleja en todos los rangos de edad y niveles educativos? La información relativa al primer criterio de clasificación se presenta en el cuadro 3, del cual se pueden destacar tres elementos principales: en primer término, que con excepción de la población de 16 a 25 ańos, en todos los grupos se presentaron aumentos de participación, ${ }^{14}$ en segundo lugar, el mayor dinamismo se registró en los grupos de 36 a 45 y de 46 a 55 ańos, donde el primero se consolida como el rango de edad con la mayor presencia de mujeres, mientras que el segundo es el que presenta el mayor dinamismo, hecho que sin duda es un dato notable pues parece indicar que durante este periodo las mujeres de este grupo deciden participar más activamente en el mercado laboral.

Los factores que llevan a este comportamiento pueden ser diversos y seguramente exceden el objetivo de este trabajo, pero aspectos como la caída del poder adquisitivo, el incremento de la inestabilidad e incertidumbre laboral pueden estar presentes. Finalmente, el tercer elemento a

\footnotetext{
${ }^{13}$ Para tener mayor conocimiento sobre la tendencia de la participación de la mujer en el mercado laboral, así como sus causas véase Loggia-Gago (1998), García y Oliveira (1990a y 1990b, 1994), Rendón (2003) y Castro-Lugo (2005).

${ }^{14}$ Este comportamiento es distinto al que se observaba en los setenta donde las mujeres del grupo de edad más joven eran las que presentaban la mayor participación, Loggia-Gago (1998).
} 
destacar es la tendencia hacia una mayor convergencia en los niveles de participación en los distintos rangos de edad, lo que parece indicar una mayor estabilidad de las mujeres dentro de su trayectoria laboral.

\section{Cuadro 3 \\ Contribución de mujeres en el mercado laboral por grupo de edad, 2000-2004. Porcentaje}

\begin{tabular}{lccccc}
\hline Año & $16-25$ & $26-35$ & $36-45$ & $46-55$ & $56-65$ \\
\hline 2000 & 40.0 & 37.3 & 40.7 & 34.6 & 28.1 \\
2001 & 40.7 & 38.6 & 41.4 & 35.2 & 27.6 \\
2002 & 39.4 & 40.1 & 39.7 & 36.1 & 30.4 \\
2003 & 38.5 & 38.8 & 41.7 & 36.3 & 28.0 \\
2004 & 38.6 & 39.8 & 41.7 & 39.0 & 31.0 \\
\hline
\end{tabular}

Nota: información considerando las 32 ciudades o áreas metropolitanas.

Fuente: elaboración propia a partir de ENEU, varios años.

\section{Cuadro 4}

Contribución de mujeres en el mercado laboral por nivel educativo, 2000-2004. Porcentaje

\begin{tabular}{ccccccc}
\hline Año & Sin instrucción & Primaria & Secundaria & Preparatoria & Universidad & Posgrado \\
\hline 2000 & 36.1 & 34.5 & 40.3 & 36.5 & 39.7 & 33.0 \\
2001 & 37.7 & 35.4 & 39.9 & 39.8 & 40.8 & 35.7 \\
2002 & 36.5 & 35.6 & 40.2 & 38.7 & 41.5 & 36.0 \\
2003 & 36.4 & 35.0 & 39.5 & 38.3 & 41.1 & 39.9 \\
2004 & 38.5 & 36.2 & 39.5 & 37.9 & 43.6 & 39.0 \\
\hline
\end{tabular}

Nota: información considerando las 32 ciudades o áreas metropolitanas.

Fuente: elaboración propia a partir de ENEU, varios ańos.

Por su parte, el cuadro 4 presenta la contribución de las mujeres en el mercado laboral por nivel educativo. Dentro de los elementos más relevantes se encuentra un comportamiento desigual por grados de escolaridad, aunque en general todos los niveles, con excepción de secundaria, aumentan su contribución.

A partir de lo anterior, se establece que el incremento de su participación laboral se dio tanto en trabajos calificados (universidad y posgrado) como no calificados (sin instrucción y primaria), hecho que parece indicar que la mayor presencia de mujeres en el mercado laboral no responde únicamente a la existencia de niveles educativos más elevados, y con ello 
un mayor costo de oportunidad por quedarse en casa, sino también a la necesidad de contribuir al ingreso del hogar o en su caso de asumir la responsabilidad de jefatura de hogar. ${ }^{15}$

Un elemento adicional a señalar, al igual que en el caso de los rangos de edad, es que en el periodo se aprecia una tendencia a la convergencia en los grados de participación por niveles educativos.

$¿$ La creciente presencia de las mujeres dentro del mercado laboral puede ser indicativo de mayor demanda de este tipo de trabajo y, por tanto, de mejores remuneraciones relativas? La grafica I presenta los datos sobre el salario relativo promedio de las mujeres en relación al de los hombres, en ella se puede apreciar que existe una diferencia permanente de remuneraciones favorable a los hombres y representa aproximadamente $20 \%$, aunque parece mejorar ligeramente, pero debemos reconocer que es un periodo corto como para derivar conclusiones más generales.

\section{Gráfica I}

Salario promedio relativo de las mujeres en relación a los hombres, 2000-2003. Porcentaje

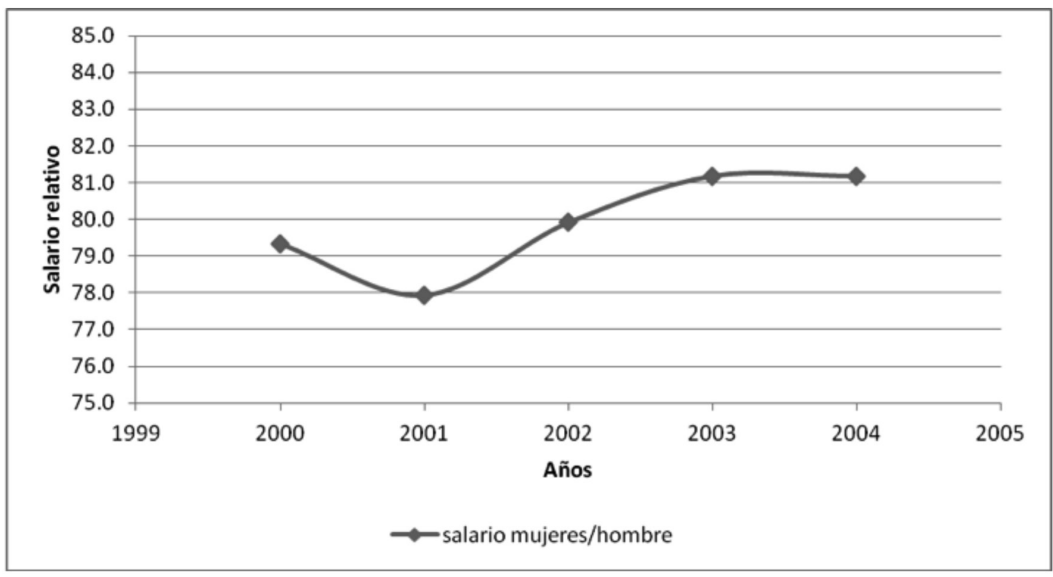

Nota: información considerando las 32 ciudades o áreas metropolitanas.

Fuente: elaboración propia a partir de ENEU, varios ańos.

¿Estas diferencias se mantienen considerando criterios como edad y niveles educativos?, factores relevantes en la formación de capital humano.

$\mathrm{Al}$ respecto, el cuadro 5 presenta referencias sobre los salarios relativos por sexo y grupo de edad. La información que aporta este cuadro permite establecer que persistentemente las mujeres, en promedio, reciben re-

\footnotetext{
${ }^{15}$ De acuerdo con el Censo de Población 2010, 24.5\% de los hogares tiene como jefe de familia a una mujer.
} 


\section{Cuadro 5}

Salario de las mujeres con relación a los hombres por grupo de edad, 2000-2004. Porcentaje

\begin{tabular}{rccccc}
\hline Año & $16-25$ & $26-35$ & $36-45$ & $46-55$ & $56-65$ \\
\hline 2000 & 91.5 & 87.1 & 69.9 & 71.6 & 68.4 \\
2001 & 92.0 & 81.7 & 68.9 & 73.3 & 75.3 \\
2002 & 92.8 & 84.1 & 73.6 & 71.2 & 69.8 \\
2003 & 91.1 & 84.8 & 75.7 & 73.1 & 73.0 \\
2004 & 92.9 & 81.8 & 78.2 & 73.9 & 67.2 \\
\hline
\end{tabular}

Nota: información considerando las 32 ciudades o áreas metropolitanas.

Fuente: elaboración propia a partir de ENEU, varios años.

muneraciones inferiores a las de los hombres del mismo grupo demográfico, y estas diferencias varían por año y rango de edad, desde $7.1 \%$ hasta 32.8 por ciento.

Con relación a su evolución durante el periodo de análisis se establece, en términos generales, que parece existir una orientación hacia la reducción de las diferencias salariales, aunque en su trayecto no se aprecia una tendencia definitiva, dado que se presentan retrocesos y avances, pero sí se puede señalar que en general el valor promedio es superior a los registrados en los años iniciales.

Finalmente, si nos preguntamos: ¿existen algunos rangos de edad con mayores diferencias salariales por sexo?, la respuesta, a partir de la información que proporciona el cuadro 5, sería afirmativa; pero además, un dato interesante es que la desventaja salarial femenina parece aumentar con la edad, dado que el salario relativo promedio para todos los años así lo indican, al pasar de 7.9\% de desventaja para el grupo más joven hasta $29.2 \%$ para el de mayor edad.

Esta mayor diferencia salarial por género en la medida que se incrementa la edad puede estar indicando un incremento en la brecha de experiencia, dado que al inicio de la vida productiva, tanto hombres como mujeres disponen de pocos antecedentes de trabajo, pero a lo largo del lapso laboral, los hombres mantienen una continuidad que les otorga mayor experiencia relativa frente a las mujeres que pueden tener una participación laboral más discontinua, lo cual se reflejará en menor salario relativo, aunque posiblemente no sea la única causa asociada con la creciente desventaja remunerativa.

La tendencia a elevar los niveles de participación laboral de las mujeres en los diferentes rangos de edad, así como una destacada presencia de ellas entre los rangos de más de 45 años, permiten suponer que en el 
largo plazo las diferencias remunerativas por sexo, asociadas a la experiencia, tenderían a disminuir.

La información anterior, si bien indica diferencias por rango de edad, puede no ser razón suficiente para establecer la presencia de discriminación salarial, dado que se comparan remuneraciones promedio por sexo para grupos de edad similares, que de alguna manera pudieran expresar iguales niveles de experiencia, aunque como comentamos líneas arriba, igual edad no necesariamente implica similar experiencia; además, no se sabe cómo es la formación educativa de cada grupo, por ello a continuación exploramos sobre los salarios relativos considerando niveles de escolaridad, los cuales se presentan en el cuadro 6 .

\section{Cuadro 6 \\ Salario de las mujeres con relación al de los hombres por nivel educativo, 2000-2004. Porcentaje}

\begin{tabular}{ccccccc}
\hline Año & Sin instrucción & Primaria & Secundaria & Preparatoria & Universidad & Posgrado \\
\hline 2000 & 65.4 & 73.8 & 88.0 & 80.8 & 71.6 & 65.5 \\
2001 & 68.3 & 74.4 & 89.5 & 80.2 & 68.2 & 63.6 \\
2002 & 69.0 & 77.6 & 89.7 & 78.9 & 70.4 & 69.0 \\
2003 & 65.2 & 74.5 & 90.3 & 82.5 & 70.5 & 69.3 \\
2004 & 67.0 & 75.7 & 89.2 & 82.1 & 70.3 & 70.3 \\
\hline
\end{tabular}

Nota: información considerando las 32 ciudades o áreas metropolitanas.

Fuente: elaboración propia a partir de ENEu, varios ańos.

Bajo esta clasificación, al igual que la anterior, existen diferencias salariales relativas a favor de los hombres con rangos que van desde $10.7 \%$, para los de secundaria, hasta 33\% para los que se encuentran sin instrucción escolar.

El ordenamiento de los trabajadores por niveles de instrucción escolar hace pensar que se trata de población más homogénea en lo que se refiere a su dotación de capital humano; sin embargo, en este caso, aun con semejante nivel educativo se desconoce la dotación de experiencia que disponen unos y otros trabajadores, y que puede considerarse como el otro componente del capital humano.

Algunos elementos adicionales que se destacan de la información proporcionada en el cuadro 6 son que no parecen existir, al menos a simple vista, señales claras que indiquen que las diferencias salariales tienden a reducirse en el tiempo, además las mayores desventajas se pre- 
sentan en los niveles con menor y mayor instrucción, ${ }^{16}$ hecho que resulta interesante, si pensamos que la educación constituye uno de los elementos más importantes para reducir la desigualdad de ingresos, dado su relevancia en la formación de capital humano; no obstante, en los niveles universitarios y posgrado el salario promedio de los hombres supera en más de $25 \%$ el de las mujeres, indicando que bajo el supuesto de ausencia de discriminación, la diferencia de experiencia y de otros factores adicionales es de esa magnitud.

Los resultados anteriores, en general, muestran la presencia de diferencias salariales por género que se expresan tanto a nivel de grupos de edad como por niveles educativos, aspectos que sin duda son relevantes y constituyen un primer resultado, en el sentido de confirmar la existencia de diferencias salariales y cómo estas desigualdades resultan inaceptables para unos grupos de edad y niveles de escolaridad; sin embargo, debemos determinar en qué medida esta disparidad está sustentada en elementos objetivos, asociados con las características de los grupos que impliquen diferencias de productividad y, por tanto, en remuneraciones. Tarea que es abordada en el siguiente apartado.

\subsection{Diferencial de salarios y descomposición}

Una vez hecha una breve revisión de las características del mercado laboral femenino, donde se identificaron disparidades salariales por grupos de edad y niveles de escolaridad por sexo, se procede a aplicar la metodología de Oaxaca-Blinder con la finalidad de determinar la magnitud de las diferencias salariales y la proporción de estas desigualdades no asociadas a las características de los grupos, mismas que pueden considerarse como discriminación salarial.

Un primer paso para el cálculo de la discriminación es la estimación de las ecuaciones salariales por género y año, donde se incorpora la educación por años de escolaridad, así como la experiencia potencial y se introduce un control para las personas con jornadas laborales completas, esto con el objetivo de identificar si las remuneraciones por hora difieren entre los que tienen jornadas completas o no, ${ }^{17} \mathrm{y}$ finalmente, se incorpora el factor de expansión, lo que arroja una población para varones de entre 5.67 y 5.28 millones, y 3.55 y 3.43 millones para mujeres, en los diferentes años.

\footnotetext{
${ }^{16}$ Este comportamiento puede estar indicando la presencia de piso pegajoso y techo de cristal tal como lo documentan Arceo y Campos (2013).

${ }^{17}$ A partir de 40 horas se consideró como jornada completa.
} 
Los resultados de las estimaciones salariales indican que los coeficientes presentan el signo esperado, y en todos los casos son significativos. ${ }^{18}$ Dentro de los aspectos a destacar de las estimaciones salariales se puede señalar que el piso salarial, captado por la constante, y que se entiende como el ingreso que recibe un(a) trabajador(a) con cero años de escolaridad, sin experiencia y sin jornada laboral competa, el de los hombres es mayor que el de las mujeres, por otra parte el rendimiento educativo por año de escolaridad permanentemente es superior en las mujeres $(10 \%$ aproximadamente) frente a los hombres ( $9 \%$ aproximadamente).

Mientras que en lo relativo a la experiencia se presenta el caso contrario, hecho que puede explicar porqué la brecha salarial por sexo se amplía conforme aumenta el rango de edad de los trabajadores, tal como se aprecia en el cuadro 5. Finalmente, trabajar una jornada completa sí presenta diferencias respecto a los que no lo hacen y ésta es mayor en hombres que en mujeres. En general la capacidad explicativa del modelo, expresado mediante el indicador $\mathrm{R}^{2}$ ajustado, está en niveles convencionales, 0.37 para hombres y 0.40 en mujeres.

Un segundo paso es calcular el salario promedio por sexo, el cual se expresa como el logaritmo del salario mensual en dólares de 2000, condicionado por la media de la distribución, los resultados se presentan en las primeras dos filas del cuadro 7 , donde se aprecia una diferencia salarial entre ambos sexos a favor del hombre en cada uno de los ańos analizados (fila 3), la cual varía sobre $20 \%$, que es similar a lo observado en la gráfica I.

La descomposición de las diferencias en términos absolutos se presenta en las filas 4 y 5 , la primera correspondiente a la parte asociada con los atributos y, la segunda, aquella no relacionada con las características; es decir, la disparidad no explicada, misma que es considerada como discriminación salarial, mientras que los términos relativos se presentan en las filas 6 y 7 en el mismo orden.

Los resultados en el cuadro 7 permiten establecer, que no sólo existe una diferencia salarial por sexo de aproximadamente $20 \%$ a favor de los hombres y que se mantiene estable durante el periodo de análisis, sino que la mayor parte de las brechas se deben a la porción no explicada por los atributos de capital humano, la cual puede vincularse con la discriminación salarial por sexo.

Así, se puede establecer que en promedio la discriminación salarial, para las 32 ciudades (seis regiones) que se incluyen en el análisis, en contra de las mujeres, equivale a $17 \%$ del salario. En el 2000 la parte no

\footnotetext{
${ }^{18}$ Por motivos de espacio no se incluyen los resultados de las estimaciones salariales en el documento pero están a la disposición de quien lo solicite.
} 


\section{Cuadro 7 \\ Descomposición del diferencial de salarios por sexo en México, 2000-2004}

\begin{tabular}{|c|c|c|c|c|c|c|}
\hline & Indicadores & 2000 & 2001 & 2002 & 2003 & 2004 \\
\hline 1 & Salario promedio masculino* & 5.752 & 5.828 & 5.906 & 5.978 & 5.951 \\
\hline 2 & Salario promedio femenino* & 5.549 & 5.618 & 5.704 & 5.788 & 5.757 \\
\hline 3 & Diferencia salarial & 0.203 & 0.210 & 0.202 & 0.190 & 0.194 \\
\hline 4 & Atributos & 0.031 & 0.040 & 0.028 & 0.014 & 0.025 \\
\hline \multirow[t]{2}{*}{5} & Discriminación absoluta & 0.172 & 0.170 & 0.174 & 0.176 & 0.169 \\
\hline & Discriminación relativa & & & & & \\
\hline 6 & \% Explicado (atributos) & 15.2 & 18.9 & 13.7 & 7.5 & 13.1 \\
\hline 7 & \% No explicado (discriminación & 84.8 & 81.1 & 86.3 & 92.5 & 86.9 \\
\hline 8 & Total & 100.0 & 100.0 & 100.0 & 100.0 & 100.0 \\
\hline
\end{tabular}

* Salario promedio mensual en dólares del 2000.

Nota: información considerando las 32 ciudades o áreas metropolitanas.

Fuente: elaboración propia a partir de ENEU, varios ańos.

explicada del diferencial es de $84.8 \%$, proporción que alcanzó su máximo en el 2003, con lo que se puede establecer una tendencia creciente.

Estos resultados, sobre la proporción de la parte no explicada de la desigualdad salarial, coinciden con los obtenidos por Hernández-Martínez (1995), Rica y Ugidos (1995) y García y Morales (2006) para el mercado laboral español, donde el porcentaje atribuido a la parte no explicada se encuentra en los márgenes señalados aquí, es decir, superiores a 70\%. En los trabajos citados para el caso de Argentina, Di Paola y Berges (1997) y Di Pasquale y Atucha (2003) igualmente obtienen resultados que son comparables en el sentido de que la mayor parte de las diferencias salariales por sexo no están asociadas con distintas dotaciones de factores entre ellos, pero también encuentran correspondencia en los trabajos de México, como es el caso de Pagán y Ullibarri (2000); Martínez-Jasso y Acevedo-Flores (2004), y Rodríguez-Pérez y CamberosCastro (2007), Popli (2008); Cacciamani y Tatei (2013); Arceo y Campos (2013); y Rodríguez-Pérez y Castro-Lugo (2014).

A partir de lo anterior se puede afirmar que aun cuando el diferencial salarial total por sexo se mantiene relativamente estable, alrededor de $20 \%$, la participación de la discriminación salarial dentro del diferencial sí aumentó; por lo tanto, se puede decir que durante el periodo 20002004, en el mercado laboral de México existe discriminación salarial en contra de las mujeres que laboran en actividades remuneradas, y que de acuerdo con las estimaciones realizadas a partir de la metodología con- 
vencional, el nivel de discriminación salarial absoluta es de aproximadamente 17 por ciento.

\section{Discriminación salarial regional}

Como se observó, la discriminación toma presencia en todos los años analizados; sin embargo, es razonable preguntar: ¿este fenómeno se expresa de manera homogénea en México o será diferente por regiones?, para dar respuesta a esta pregunta a continuación se presenta el análisis en seis regiones del país, con el objetivo de conocer el efecto espacial del fenómeno de la discriminación en México.

Para esto se muestra la disparidad salarial total por sexo, misma que se presenta en la gráfica II, de la cual se pueden destacar, fundamentalmente dos elementos, el primero, además de la existencia de diferencias en las remuneraciones promedio por género para todas las regiones, las disparidades salariales son significativas pues la mayor magnitud se observa para el centro, la cual se ubica en aproximadamente $25 \%$, mientras la capital muestra niveles de desigualdad 10 puntos porcentuales inferiores a éstos; lo que permite establecer que la disparidad salarial entre regiones es significativa y no podemos hablar de homogeneidad.

\section{Gráfica II}

Disparidad salarial total por sexo y por regiones, 2000-2004

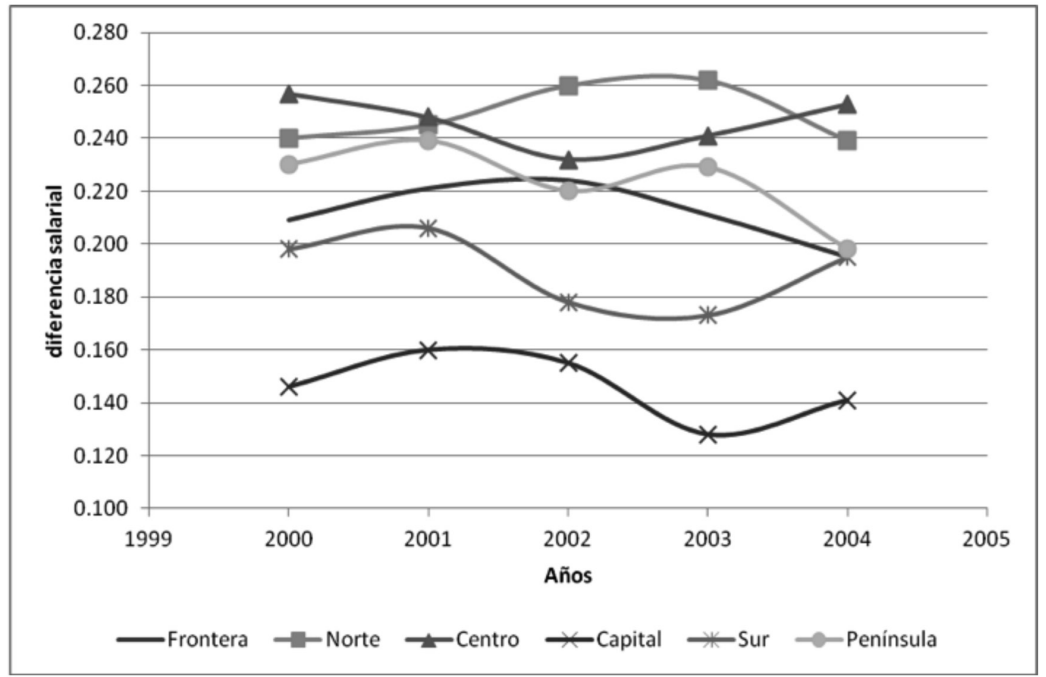

Nota: información considerando las 32 ciudades o áreas metropolitanas.

Fuente: elaboración propia a partir de ENEU, varios ańos. 
El otro elemento a señalar es el hecho de que prácticamente no se presentan cambios en el orden por regiones, indicando que los niveles de desigualdad existentes dentro de éstas se vinculan con elementos estructurales, dado que permanecen en el tiempo y mantienen un orden. Con excepción de la península, no se aprecia una tendencia que indique una reducción de las disparidades salariales o hacia la convergencia entre ellas.

Una vez documentada la disparidad salarial regional, y antes de comentar los resultados de la descomposición salarial, es pertinente mencionar, de manera general, sobre algunos aspectos que se pueden destacar de las estimaciones salariales por regiones, ${ }^{19}$ donde al igual que a nivel agregado en todas las regiones y años, los coeficientes presentan el signo esperado y son significativos a $99 \%$ de grado de confianza.

En primer término, destacar que las mayores remuneraciones de la frontera no se vinculan con las tasas de rendimiento educativo ni con la retribución a la experiencia (aspectos que se ubican por debajo del promedio regional), sino con el elevado piso salarial que es superior al resto de las regiones, esto se cumple para ambos sexos; el segundo elemento es que la capital presenta los rendimientos educativos más elevados, pero la remuneración a la experiencia es inferior al promedio, mientras que el piso salarial puede considerarse elevado.

Por otra parte, las regiones norte y península presentan su mayor rendimiento en la experiencia y el sur tiene desventaja relativa en las remuneraciones iniciales, (piso salarial) especialmente en mujeres, mientras los rendimientos educativos de los hombres son inferiores al promedio: (8\%) y en mujeres se ubica en 11 por ciento.

Finalmente es pertinente destacar la existencia de diferencias en los rendimientos al capital humano por sexo en las distintas regiones, aunque cada una de ellas muestra patrones particulares.

Los resultados de las ecuaciones salariales permiten establecer que las diferencias de salario entre regiones no sólo están asociadas con desigualdades en los rendimientos educativos o experiencia, sino también en lo relativo al nivel de remuneraciones iniciales, asimismo, se expresan diferencias importantes por sexo al interior de las regiones.

A partir de las estimaciones se procedió a la segmentación de las diferencias salariales por sexo dentro de las regiones, a partir de ello se determinan dos elementos: primero, la parte de la inequidad de ingresos no asociada con las características del capital humano, es decir, la propor-

${ }^{19}$ Los resultados de estas ecuaciones salariales no se presentan en el documento por motivos de espacio pero están a la disposición de quien la solicite. 
ción del total no explicada y vinculada con la discriminación, y el segundo, el nivel de desigualdad salarial asociado con la discriminación.

La grafica III presenta el primer elemento, donde se destacan fundamentalmente tres aspectos: i) la parte no explicada de la desigualdad total por sexo es elevada y en todas las regiones/año representa más de $70 \%$, ii) en algunas regiones y años (sur y capital) el porcentaje es superior a $100 \%$, lo que implica que de acuerdo con las características laborales promedio, mismas que se reflejarían en la productividad, las mujeres, en ausencia de discriminación, deberían tener una remuneración promedio superior a la de los hombres, por tanto, en estos casos la discriminación es mayor que la desigualdad salarial total y, finalmente iii) en general parece apreciarse una tendencia hacia la homogenización regional del porcentaje de la desigualdad no asociada a características laborales, aunque ésta es hacia mayores niveles, lo que indicaría que para todas las regiones, una mayor proporción de la desigualdad salarial por sexo está vinculada con la discriminación, en este sentido, las modificaciones o diferencias en la estructura económica de las regiones no parecen reflejarse en la discriminación salarial por género.

\section{Gráfica III}

\section{Proporción de la desigualdad salarial por sexo no explicada, por regiones, 2000-2004. Porcentaje}

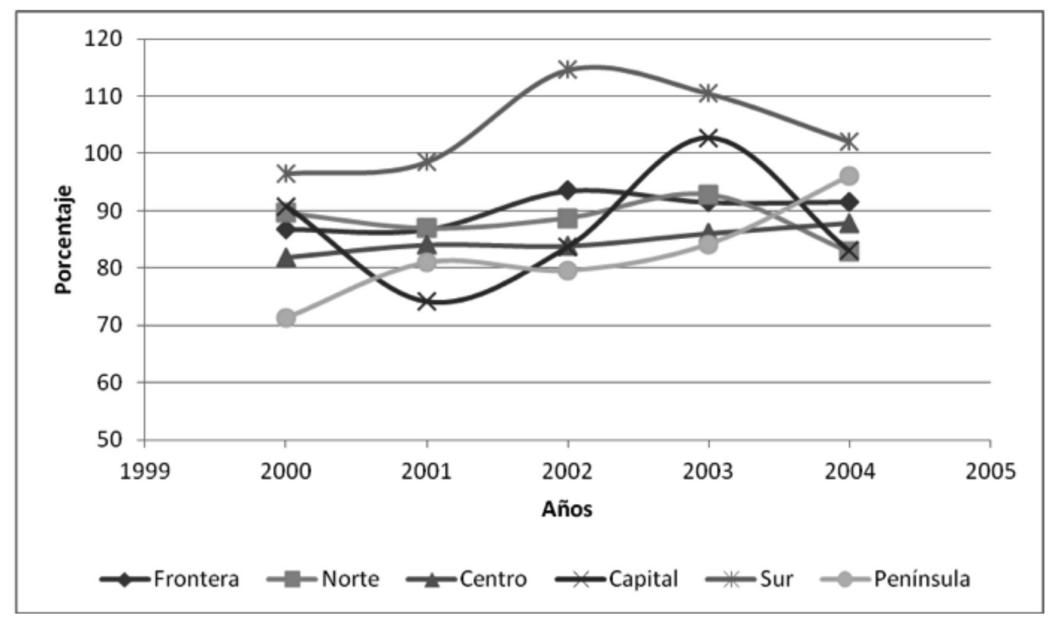

Nota: información considerando las 32 ciudades o áreas metropolitanas.

Fuente: elaboración propia a partir de ENEU, varios años.

La información sobre el nivel de desigualdad salarial regional asociado con la discriminación se presenta en la gráfica Iv, donde se aprecia que al inicio del periodo la magnitud de la discriminación es heterogénea, con 
niveles entre $13.7 \%$ para la capital, y $22.2 \%$ para el norte; a lo largo del lapso de análisis se aprecian variaciones relevantes entre regiones pero al fin se estima una tendencia hacia la convergencia alrededor de $20.0 \%$, con excepción de la capital que se ubica en niveles inferiores, mostrando diferencias importantes con el resto de las regiones.

A partir de este comportamiento se puede establecer que si se eliminara la discriminación salarial por género, la desigualdad en las remuneraciones se reduciría significativamente.

\section{Gráfica IV}

\section{Discriminación salarial absoluta por regiones, 2000-2004}

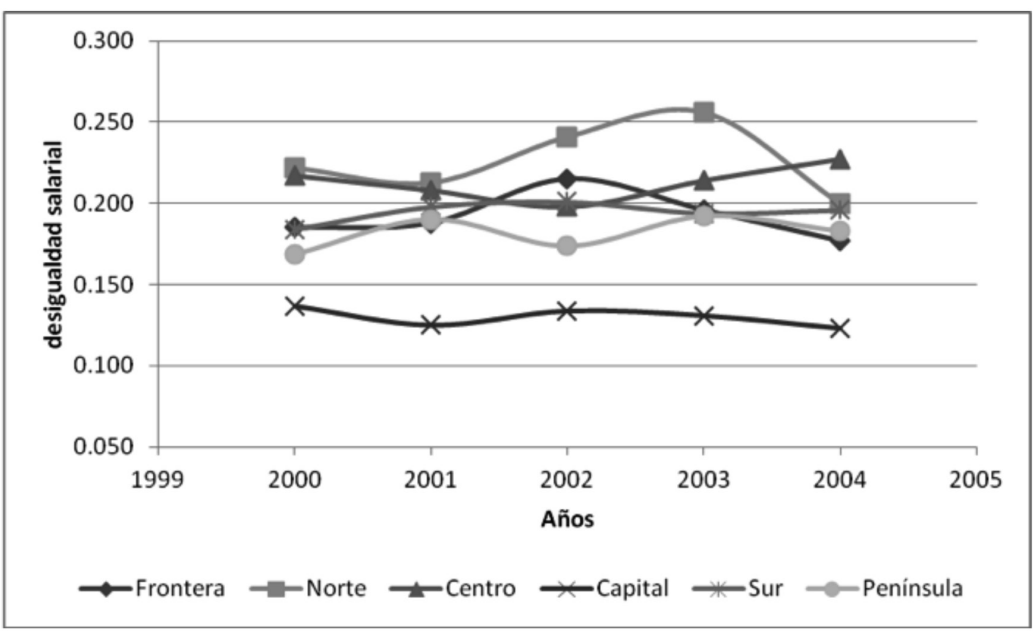

Nota: información considerando 32 ciudades o áreas metropolitanas.

Fuente: elaboración propia a partir de ENEU, varios años.

\section{Conclusiones}

El estudio de la desigualdad salarial por sexo, así como la exploración y medición de la magnitud de ésta, asociada con la discriminación salarial, es un tema que ha captado la atención de los estudiosos del mercado laboral en distintos países, y los resultados indican que, no obstante la utilización de diferentes fuentes de información y periodos de tiempo, persiste la presencia de discriminación salarial, donde México no es la excepción y los estudios previos así lo muestran.

En las últimas décadas, la creciente participación de las mujeres en el mercado laboral es una de las características más relevantes, no obstante esta mayor presencia, permanecen las diferencias salariales por sexo, las cuales se mantienen por niveles educativos y se incrementan con la edad. 
La estimación de las ecuaciones salariales permitieron identificar diferencias salariales del orden de $20 \%$ a favor de los hombres y la posterior descomposición del ingreso confirmaron que más de $85 \%$ de estas diferencias están asociadas con la discriminación, dado que son desigualdades no atribuibles a las dotaciones de capital humano.

Esta proporción no explicada es comparable con los niveles obtenidos en estudios realizados en otros países, así como con algunos que se hicieron en México. Por regiones se aprecia un comportamiento heterogéneo donde las diferencias oscilan entre $15 \%$ y $25 \%$, con niveles de discriminación que tienden a converger en $20 \%$, con excepción de la región capital que se ubica sobre 13 por ciento.

A partir de lo anterior es posible sostener que existe un espacio para la implementación de políticas que fomenten una mayor igualdad de género en las remuneraciones, dado que la eliminación de la discriminación reduciría la desigualdad salarial en aproximadamente $85 \%$; por otra parte, los indicadores de desigualdad y discriminación salarial por regiones no parecen presentar diferencias importantes, no obstante que la apertura económica imprimió una diferenciación en las estructuras económicas regionales.

\section{Bibliografía}

Aguayo-Tellez, Ernesto (2011), "The impact of trade liberalization policies and FDI on gender inequalities: a literature review", World Development Report 2012, Gender Equality and Development, The World Bank, New York, pp. 1-34.

Alarcón, Diana y Terry McKinley (1994), "Gender differences in wages and human capital: case study of female and male urban workers in Mexico from 1984 to 1992", Frontera Norte, 6 (12), El Colegio de la Frontera Norte, Tijuana, pp. 41-50.

Albrecht, James, Aico Van Vuuren y Susan Vroman (2009), "Counterfactual distributions whith sample selection adjuntments: econometric theory and an application to the Netherlands", Labour Economics, 16 (4), Elselvier, Amsterdam, pp. 383-396.

Arceo, Eva y Raymundo Campos (2013), "Evolución de la brecha salarial de género en México", documento de trabajo núm. VII-2013, Centro de Estudios Económicos-El Colegio de México, México, pp. 1-38. 
Artecona, Raquel y Wendy Cunningham (2002), "Effects of trade liberalization on the gender wage gap in Mexico", working paper núm. 34144, The World Bank, New York, pp. 1-28.

Autor, David (2003), "Lecture note: the economics of discriminationtheory”, Graduate Labor Economics I, Massachusetts Institute of Technology, Cambridge, pp. 1-18.

Becker, Gary Stanley (1971), The Economics of Discrimination, The University of Chicago Press, Chicago.

Blinder, Alan (1973), "Wage discrimination: reduced forms and structural estimates", Journal of Human Resources, 8 (4), The University of Texas at Austin, Austin, pp. 436-455.

Brown, Cynthia, José Pagán y Eduardo Rodríguez-Oreggia (1999), "Occupational attainment and gender earning differentials in Mexico", Industrial and Labor Relations Review, 53 (1), Cornell University, New York, pp. 123-135.

Brown, Flor y Lilia Domínguez (2007), "Determinants of wage differentials in the maquila industry in Mexico: a gender perspective", working paper núm. 07-6, The International Working Group on Gender-Macroeconomics-International Economics, Salt Lake City, pp. 1-24.

Cacciamali, María Cristina y Fábio Tatei (2013), "Genero y salarios de la fuerza de trabajo calificada en Brasil y México", Problemas del Desarrollo, 172 (44), unam, México, pp. 53-79.

Castro-Lugo, David (2005), "Salarios y desigualdad territorial en las áreas urbanas de México, 1992-2002”, tesis de doctorado, Universidad Autónoma de Barcelona, Barcelona.

Castro-Lugo David, Luis Huesca-Reynoso y Nathalia Zamarrón-Otzuca (2014), "Discriminación salarial por género en la industria manufacturera de la frontera norte de México en el periodo 20052011", Revista Nóesis, 23 (45), Universidad Autónoma de Ciudad Juárez, Ciudad Juárez, en prensa.

Cheng, Zhiming, Fei Guo, Hugo Graeme y Yuan Xin (2013), "Employment and wage discrimination in the chinese cities: a compara- 
tive study of migrants and locals", Habitat International, 39, Elselvier, Amsterdam, pp. 246-255.

Chi, Wei, Li Bo y Yu Qiumei (2007), "Decomposition of changes in earnings inequality in China: a distributional approach", working paper no. 3806, Labor and Demographic Economics, Tsinghua University, Beijing, pp. 1-34.

Di Paola, Rosángela y Miriam Berges (1997), "Sesgo de selección y estimación de la brecha por género para Mar de la Plata”, Nülan, Universidad Nacional de Mar de la Plata, La Plata, pp. 1-15.

Di Pasquale, Actis Eugenio y Ana Julia Atucha (2003), "Brechas salariales: discriminación o diferencias de productividad", Momento Económico, núm. 126, unam, México, pp. 23-33.

Domínguez, Lilia y Flor Brown (2010), "Trade liberalization and gender wage inequality in Mexico", Feminist Economics, 16 (4), Routledge, Philadelphia, pp. 53-79.

El Hamidi, Fatma (2008), "Trade liberalization, gender segmentation, and wage discrimination: evidence from Egypt", Economic Research Forum, working paper no. 414, University of Pittsburgh, Pittsburgh, pp. 1-26.

Fuentes, Jeanette, Amalia Palma y Rodrigo Montero (2005), "Discriminación salarial por género en Chile: una mirada global”, Estudios de Economía, 32 (2), Universidad de Chile, Santiago, pp. 133-157.

García, Brígida y Orlandina de Oliveira (1990a), "Expansión del trabajo femenino y transformación social en México", en Fernando Alberto Cortés Cáceres (coord.), México en el umbral del milenio, El Colegio de México, México, pp. 345-374.

García, Brígida y Orlandina de Oliveira (1990b), "El trabajo femenino en México a finales de los ochenta", en Elia Ramírez-Bautista e Hilda Dávila-Ibañez (coords.) Trabajo femenino y crisis en México: tendencias y transformaciones actuales, Universidad Autónoma Metropolitana unidad Xochimilco, México, pp. 53-78.

García, Brígida y Orlandina de Oliveira (1994), Trabajo femenino y vida familiar en México, El Colegio de México-Centro de Estudios Demográficos y de Desarrollo Urbano, México. 
García, José Ignacio y María Dolores Morales (2006), “Discriminación salarial en el mercado de trabajo español con especial referencia al caso de Andalucía”, documento de trabajo E2006/18, Universidad de la Rioja, Logrońo, pp. 1-36.

Garro-Bordonaro, Nora y Eduardo Rodríguez-Oreggia (1995), “Discriminación salarial y segregación ocupacional. Las mujeres asalariadas en México, 1987-1993", Análisis Económico, 12 (27), Universidad Autónoma Metropolitana unidad Azcapotzalco, México, pp. 105-126.

Hanson, Gordon (2003), "What has happened to wages in México since NAFTA? Implications for hemispheric free trade", working paper series no. 9563, National Bureau of Economic Research, Cambridge, pp.1-43.

Hernández-Martínez, Pedro (1995), “Análisis empírico de la discriminación salarial de la mujer en España”, Investigaciones Económicas, 19 (2), Fundación SEPI, Madrid, pp. 195-215.

INEGI (Instituto Nacional de Estadística y Geografía) (2000-2004), Encuesta Nacional de Empleo Urbano (ENEU), INEgi, Aguascalientes, México.

INEGI (Instituto Nacional de Estadística y Geografía) (2009), Mujeres y hombres en México, 2009, INEGI-Imujer, Aguascalientes.

INEGI (Instituto Nacional de Estadística y Geografía) (2010), Censo de Población 2010. INEGI, México.

Loggia-Gago, Silvia María (1998), “Legislación laboral y participación de la mujer en el mercado de trabajo. El caso de Suecia y México: un análisis comparativo", Papeles de Población, 15 (4), Universidad Autónoma del Estado de México-Centro de Investigación y Estudios Avanzados de la Población, Toluca, pp. 95-125.

Martínez-Jasso, Irma y Gloria Acevedo-Flores (2004), "La brecha salarial en México con enfoque de género: capital humano, discriminación y selección muestral”, Ciencia, 7 (1), Universidad Autónoma de Nuevo León, Monterrey, pp. 66-71. 
McConnell, Campbell, Stanley Bruce y David McPherson (2003), Economía laboral, McGraw-Hill-Interamericana de España, Barcelona.

Menon, Nidhiya y Yana van der Meulen Rodgers (2009), "International trade and the gender wage gap: new evidences fron India's manufacturing sector", World Development, 37 (5), Elselvier, Amsterdam, pp. 965-981.

Mincer, Jacob (1974), Schooling, experience, and earnings, National Bureau of Economic Research-Columbia University Press, New York.

Montero, Rodrigo y Paz Garcés (2009), “¿Existe discriminación salarial contra la población indígena en Chile?”, El Trimestre Económico, 76 (3), Fondo de Cultura Económica, México, pp. 645-669.

Neumark, David (1988), “Employers' discriminatory behavior and the estimation of wage discrimination", The Journal of Human Resources, 3 (23), The University of Texas at Austin, Austin, pp. 279-295.

Oaxaca, Ronald (1973), "Male-female wage differentials in urban labour markets", International Economic Review, 14 (3), University of Pennsylvania-The Osaka University, Philadelphia, pp. 693-709.

Oaxaca, Ronald y Ranson Michael (1994), "On discrimanation and the descomposition of wage differentials", Journal of Econometrics, 61 (1), Elsevier, New York, pp. 5-21.

Pagán, José y Miriam Ullibarri (2000), "Group heterogeneity and the gender earning gap in Mexico", Economía Mexicana, Nueva Época, 9 (1), Centro de Investigación y Docencia Económica, México, pp. 23-40.

Popli, Gurleen (2008), "Gender wage discrimination in México: A distributional approach", Sheffeld Economic Research Paper Series, no. 2008006, University Sheffield, Sheffield, pp. 1-35.

Popli, Gurleen (2013), "Gender wage differentials in México: A distributional approach", Journal of the Royal Statistical Society: A, 176 (2), Statics in Society, London, pp. 295-319. 
Ramos, Vicente, Javier Rey-Maquieira y María Tugores (2002), “Análisis empírico de discriminación por razón de género en una economía especializada en el turismo", Annals of Tourism Research en Español, 4 (1), Universitat de les Illes Balears, Palma de Mallorca, pp. 239-258.

Rendón, Teresa (2003), Trabajo de hombres y trabajo de mujeres en el México del siglo XX, UnAM, México.

Rica, Sara de la y Arantza Ugidos (1995), “'Son las diferencias en capital humano determinantes de las diferencias salariales observadas entre hombres y mujeres?", Investigaciones Económicas, 19 (3), Fundación sePI, Madrid, pp. 395-414.

Rica, Sara de la, Juan Dolado y Vanesa Llorens (2008), "Ceiling of floors? Gender wage gaps by education in Spain”, Journal of Population Economic, 21, Springer, Berlin, pp. 751-776.

Río Coral del, Carlos Gradín y Olga Cantó (2011), "The measurement of gender wage discrimination: the distributional approach revisited", Journal of Economic Inequality, 9, Springer, Berlin, pp. 57-86.

Rodríguez-Pérez, Reyna y Mario Camberos-Castro (2007), "Análisis de la discriminación de la mujer en Hermosillo, Sonora", Revista Política y Cultura, núm. 28, Universidad Autónoma Metropolitana unidad Xochimilco, México, pp. 219-250.

Rodríguez-Pérez, Reyna y David Castro-Lugo (2014), "Análisis de la discriminación salarial en Saltillo y Hermosillo: un análisis comparativo en la industria manufacturera”, Revista Nóesis, 23 (46), Universidad Autónoma de Ciudad Juárez, Ciudad Juárez, en prensa.

Silber, Jacques y Michal Weber (1999), "Labour market discrimination: are there significant differences between the various decomposition procedures?", Applied Economics, 31 (3), Routledge, Philadelphia, pp. 359-365.

Ullibarri-Arce, Miren (2003), "Diferencias salariales entre los sectores públicos y privados por género, escolaridad y edad. El caso de 
España”, El Trimestre Económico, 70 (2), Fondo de Cultura Económica, México, pp. 233-253.

Weichselbaumer, Doris y Dudolf Winter-Ebmer (2005), "A meta-analisis of the international gender wage gap", Journal of Economic Surveys, 19 (3), Blackwell, Oxford, pp. 479-511.

Recibido: 8 de marzo de 2011. Reenviado: 22 de noviembre de 2011. Aceptado: 26 de junio de 2013.

Reyna Elizabeth Rodríguez-Pérez. Mexicana. Es doctora en ciencias por el Centro de Investigación en Alimentación y Desarrollo (CIAD). Actualmente es profesora-investigadora de la Facultad de Economía de la Universidad Autónoma de Coahuila. Miembro del Sistema Nacional de Investigadores. Su línea de investigación es economía laboral. Entre sus últimas publicaciones destacan, en coautoría: "Análisis de la discriminación salarial por género en Saltillo y Hermosillo: un estudio comparativo en la industria manufacturera", Nóesis, vol. 23, UACJ, Ciudad Juárez, pp. 219-250 (2014); en coautoría: "La calificación laboral en ocupaciones tecnológicas y no tecnológicas en México y sus regiones", Estudios Sociales, XXI (42), CIAD, Hermosillo, pp. 87-112 (2013); Desigualdad salarial como efecto del cambio tecnológico en el mercado laboral de México y sus regiones, Editorial Académica Española, Saarbrücken, 276 pp. (2012); en coautoría: "Efectos del cambio tecnológico en los mercados de trabajo regionales en México", Estudios Fronterizos, 13 (26), UABC, Mexicalli, pp. 141-174 (2012).

David Castro-Lugo. Mexicano. Doctor en economía aplicada por la Universidad Autónoma de Barcelona. Actualmente es profesor-investigador del Centro de Investigaciones Socioeconómicas (CISE) en la Universidad Autónoma de Coahuila (uAdec) y coordinador de posgrado del CISE. Miembro del Sistema Nacional de Investigadores. Líneas de investigación: mercado laboral y economía regional. Entre sus últimas publicaciones destacan, en coautoría: "La calificación laboral en ocupaciones tecnológicas y no tecnológicas en México y sus regiones", Estudios Sociales, XXI (42), CIAD, Hermosillo, pp. 87-112 (2013); en coautoría: "Cambio tecnológico y diferencia salarial en las regiones de México: un análisis de datos de panel para el sector servicios", Estudios Fronterizos, 14 (28), UABC, Mexicali, pp. 187-21 (2013); y El mercado laboral frente a las transformaciones económicas en México, (coord), Universidad Autónoma de Coahuila-Plaza y Valdés, México, 329 pp. (2014). 